\title{
Espacio de vida y entorno del barrio en adultos mayores de 65 a 74 años del área urbana de Manizales, Colombia
}

ÉDER PEÑA QUIMBAYA

> Universidad de Caldas, Grupo de investigación Gerontología y Geríatría, Manizales - Caldas, Colombia. eder.pena@ucaldas.edu.co

CARMEN LUCÍA CURCIO BORRERO

> Universidad de Caldas, Grupo de Investigaciones en Gerontología y Geriatría, Manizales - Caldas, Colombia. carmen.curcio@ucaldas. edu.co

Universidad de Valparaíso

Facultad de Arquitectura

Revista Márgenes

Espacio Arte Sociedad

Espacio de vida y entorno del barrio en adultos mayores de 65 a

74 años del área urbana de Manizales, Colombia

Diciembre 2016 Vol $13 \mathrm{~N}^{\circ} 19$

Páginas 21 a

ISSN elec. 0719-4463

ISSN imp. 0718-4034

Recepción: agosto 2016

Aceptación: noviembre 2016

\section{RESUMEN}

Según estudios realizados hasta la fecha, pocos tratan la relación espacio de vida y entorno del barrio en adultos mayores en América Latina. El objetivo de este artículo es determinar la asociación entre espacio de vida y entorno del barrio en adultos mayores de 65 a 74 años en el área urbana de Manizales, Colombia. La metodología utilizada fue un estudio cuantitativo (descriptivo, prospectivo y transversal) con una muestra de 398 adultos mayores, distribuidos en 11 comunas y 29 barrios. Se evaluó espacio de vida y entorno del barrio, se realizó un análisis uni-bivariado $(p<0,05)$. En espacio de vida hay una asociación estadísticamente significativa $(p=0,000)$ con sexo, nivel educativo e ingreso económico. También el espacio de vida hay una asociación estadísticamente significativa $(p=0,000)$ entre salir fuera del barrio y la frecuencia de 1 a 3 días a la semana; más de la mitad de adultos mayores se movieron fuera del barrio sin la ayuda de una persona. Los factores que se asociaron con espacio de vida fueron espacio de vida independiente, ingreso económico y nivel educativo. Principalmente el alto nivel educativo, espacio de vida independiente, recibir altos ingresos económicos y áreas para caminar seguro se asocian significativamente con el espacio de vida, pues favorece la movilidad de los adultos mayores en el área urbana.

PALABRAS CLAVE

espacio de vida, entorno del barrio, adultos mayores

Living space and neighborhood environment in seniors between 65 to 74 years old from the urban area of Manizales, Colombia

ABSTRACT

According to studies up to date, few research the relation between living space and neighborhood environment regarding seniors in Latin America. the objective of this article is to determine the link between living space and neighborhood environment in seniors between 65 to 74 years old in the urban area of Manizales, Colombia. The methodology that was used is a quantitative study (descriptive, prospective and transversal) with a sample of 398 seniors, distributed among 11 communes and 29 neighborhoods. The evaluation considered living space and neighborhood environment, an uni-bivariate analysis $(p<0,05)$ was conducted. On the living space there is a statistically significate link $(p=0,000)$ with gender, educational level and economical income. Also, on living space there is a a statistically significate link between going outside the neighborhood and the frequency of 1 to 3 times a week. The factors related to living space where independent living space, income and educational level. Mainly, higher educational level, independent living space, high income and safe walking areas are linked with living space, since it favors the mobility of seniors in the urban area.

KEYWORDS

living space, neighborhood environment, seniors

Espacio de vida y entorno del barrio en adultos mayores de 65 a 74 años del área urbana de Manizales, Colombia > Éder Peña Quimbaya, Carmen Lucía Curcio Borrero
Revista Márgenes N 19 Vol $13>$ Diciembre $2016>21$ Facultad de Arquitectura > Universidad de Valparaíso 


\section{INTRODUCCIÓN}

Se sabe que el grupo poblacional de adultos mayores está creciendo con rapidez en el mundo, debido al aumento de la proporción de personas de 60 años y más, con respecto al total de la población. Este envejecimiento poblacional forma parte de la transición demográfica, la cual se caracteriza por el cambio que tiene una población en el tiempo de altas a bajas tasas de mortalidad, y posteriormente el descenso sostenido de la fecundidad hasta llegar finalmente a tasas bajas de natalidad y mortalidad (Huenchuan, 2009:41). En el mundo, para el año 2025, las personas con 60 años y más serán aproximadamente 1,2 millardos y alrededor del $70 \%$ de estos adultos mayores se encontraran en los países en vía de desarrollo (OMS, 2002), en tanto para América Latina la población de adultos mayores aumentará del $8 \%$ al 15\% entre 1998 y 2025 (ONU, 2002; OMS, 2002).

En América Latina se observa que el envejecimiento de las personas mayores de 60 años, especialmente de la población total, se cuadruplicará entre 2000 y 2050 , de modo que uno de cada cuatro latinoamericanos será adulto mayor en el año 2050. Según la etapa de envejecimiento Colombia, junto con otros 14 países, se encuentra en un envejecimiento moderado con bajas tasas de fecundidad (entre 2,8 y 2,0 hijos por mujer) y un índice de envejecimiento que oscila entre el 23,2\% y el 36,9\% (Huenchuan, 2009:41).

Para Bronfenbrenner (1987:52) el entorno es el lugar en que las personas pueden interactuar cara a cara fácilmente. A medida que se envejece la relación con el entorno, puede verse afectada debido a un deterioro físico, a bajos niveles de actividad física (Frank, Kerr, Rosenberg, \& King, 2010), a un limitado desplazamiento conforme a las características que presenta el entorno como la casa, el barrio o la ciudad (Crowe, et al., 2008) y a la pérdida de las relaciones sociales (Rosso, Auchincloss, \& Michael, 2011). Lo anterior denota la importancia del entorno para la movilidad en los adultos mayores, pues ellos son particularmente cercanos a sus entornos locales (casa y barrio), especialmente cuando experimentan limitaciones en su capacidad funcional (Clarke \& Nieuwenhuijsen, 2009).

El entorno del barrio se puede investigar desde dos enfoques. El primero, el entorno construido, son las construcciones o modificaciones del entorno realizadas por el hombre en los lugares en que desarrolla su vida diaria (Renalds, Smith, \& Hale, 2010:69) tiene tres dimensiones: sistema de transporte que es la red de infraestructura física (redes de calles y transporte), patrones del uso del suelo, sea para vivienda, comercio e industrias que se distribuyen en el barrio (densidad y mezcla del suelo) y diseño urbano para las vías, tamaño y amplitud de los andenes (seguridad, atractivo y diseño del lugar) (Rosso et al., 2011:4). Para su evaluación se utiliza el sistema de información geográfico (SIG), nivel socieconómico del barrio (Raudenbush \& Sampson, 1999) y el sistema de posicionamiento global (Global Positioning System - GPS).

En el segundo enfoque se refiere a las características del barrio en el que se tienen en cuenta los elementos construidos y naturales destinados a satisfacer los intereses sociales de la comunidad (Fique, Cerón \& Rojas, 2000:13); tiene dos dimensiones: elementos construidos (Keysor, Jette \& Haley, 2005:4) y situaciones que tiene el barrio (Balfour \& Kaplan, 2002:510). Para la evaluación de las características del entorno del barrio, en cuanto a su existencia y acceso se utiliza la entrevista, debido a su fácil aplicación y bajo costo (Clarke \& Nieuwenhuijsen, 2009b). Para evaluar los elementos construidos se utiliza The Home And Community Environment instrument (HACE) (Keysor et al., 2005:39) y para las situaciones que tiene el barrio (Balfour \& Kaplan, 2002:512) se identifica los problemas sociales.

El presente estudio seguirá el segundo enfoque. Según Yen, Michael \& Perdue (2009) y Franco, et al., (2009) las características del entorno del barrio permiten establecer la relación del adulto mayor con el entorno a través de los elementos construidos como andenes, senderos, parques, bancas, parqueaderos y transporte. El estado deficiente de los elementos construidos pueden aumentar el riesgo de limitaciones funcionales a través de una menor actividad física en el barrio y permanecer un mayor tiempo en la casa (Schootman, Andresen, Wolinsky \& Malmstrom, 2006:453).

También las situaciones que se presenten en el barrio, como iluminación insuficiente, ruido excesivo, delincuencia, tráfico pesado, basuras, entre otras, pueden facilitar o restringuir la participación en actividades o la movilidad en el barrio. Varios estudios (Clarke \& Nieuwenhuijsen, 2009b; Schootman, et al., 2006; Balfour \& Kaplan, 2002) concluyen que las situaciones del barrio se asocian con la aparición de problemas sociales que afectan la movilidad en los adultos mayores.

En el espacio de vida se refleja la relación de la persona con el entorno (Allman, Sawyer, Maisiak, Sims \& Roseman, 2004). Webber, Porter \& Menec (2010:447) definen como movilidad la capacidad de moverse a sí mismo dentro de los entornos de la comunidad en que habita. Autores como Peel, et al., (2005); Baker, Bodner \& Allman (2003) y Stalvey, Owsley, Sloane \& Ball (1999) han centrado su objetivo de investigación en la movilidad que se hace en la casa y fuera de ella. Esta movilidad se evalúa a través del espacio de vida.

El espacio de vida, ha sido conceptualizado como una medida de la movilidad y de participación social, puesto que refleja el área a través de la cual se mueve una persona, desde su habitación hasta más allá de la ciudad o del país (Baker, Bodner \& Allman, 2003:1612). Autores como Peel et al. (2005); Baker, Bodner \& Allman (2003) y Stalvey et al. (1999), proponen que la evaluación del espacio de vida se debe basar en saber en qué lugares (habitación, fuera de la casa, barrio, fuera del barrio y fuera de la ciudad) ha estado la persona durante las últimas 4 semanas, la frecuencia de acceso de esos lugares y la independencia o necesidad de ayuda.

Los estudios acerca del espacio de vida en adultos mayores se han preocupado por establecer la relación entre el espacio de vida y la etnia a la cual pertenece (negros y blancos) (Allman, et al., 2004), riesgo de mortalidad (Boyle, Buchman, Barnes, James, \& Bennett, 2010), uso de dispositivos para la movilidad (Auger, et al., 2010), deterioro cognitivo (Crowe, et al., 2008), fragilidad (Li Xue, et al., 2008) y capacidad física (Peel, et al., 2005).

En América Latina no se conocen estudios que traten la relación entre espacio de vida y entorno del barrio en adultos mayores de 65 a 74 años.

El objetivo del presente estudio fue determinar la asociación entre espacio de vida y entorno del barrio en adultos mayores de 65 a 74 años área urbana de Manizales. 


\section{MÉTODOS}

Estudio transversal, prospectivo y descriptivo. Este estudio se inscribe en el proyecto International Mobility in Aging Study (IMIAS). EI estudio se lleva a cabo en cinco ciudades, dos en Canadá (Kingston, Ontario y St. Hyacinthe, Quebec), una en Brasil (Natal), una en Albania (Tirana) y una en Colombia (Manizales). Para este estudio solo se tuvieron en cuenta los datos de Manizales.

La población del estudio, de acuerdo con el censo general de 2005 comprende 24.000 ancianos (hombres y mujeres) entre 65 a 74 años que residen en el área urbana de Manizales (DANE, 2005). Se realizó un muestreo aleatorio simple y se calculó un tamaño de muestra representativo de la población de 384 ancianos entre 65 a 74 años, y para equilibrar las unidades de muestra y equiparar por sexo a conveniencia del estudio, se aproximó a 398 ancianos. Para la selección de la muestra, se tuvieron en cuenta las 11 comunas, establecidas de acuerdo con la división territorial urbana de Manizales (Instituto Geográfico Agustín Codazzi, 2009), aleatoriamente se escogieron 29 barrios y proporcionalmente se definió la muestra para cada uno de ellos (en total 198 hombres y 200 mujeres).

Los criterios de inclusión fueron: tener una edad entre 65 a 74 años, no tener deterioro cognoscitivo (contestar las preguntas de orientación del test cognoscitivo del Leganés) y aceptar-firmar el consentimiento informado.

En cuanto a los aspectos éticos, en el presente estudio se solicitó a los participantes el diligenciamiento de un consentimiento informado a través del cual se dio a conocer los objetivos, la participación libre, la confidencialidad de la información y anonimato de los participantes. Según la Resolución 8430/1993 del Ministerio de Salud de Colombia, que dicta disposiciones en materia de estudios en personas, se cataloga como de riesgo mínimo. The International Mobility in Aging Study (IMIAS) fue aprobado por los comités de ética las Universidades de Montreal y Queen's (Canadá), Universidad Federal de Rio Grande Do Norte (Brasil) y Universidad de Caldas (Colombia).

Los datos fueron registrados entre noviembre de 2011 y julio 2012, mediante entrevista personal realizada en la vivienda del anciano de acuerdo con la ubicación de la comuna, el barrio y la manzana. Se utilizó un cuestionario estandarizado, el entrenamiento para su aplicación del cuestionario tuvo como referente el manual de procedimientos del IMIAS (Gómez y Curcio, 2011).

Para establecer la presencia de deterioro cognitivo se utilizó la prueba cognitiva del Leganés (PCL) la cual ha demostrado validez de constructo (Zunzunegui, et al., 2000) y confiabilidad (García de Yébenes, Rodríguez Laso, Otero Puime, Zunzunegui \& Del Ser, 2003). Un puntaje de 4 o más.

El cuestionario de evaluación del espacio de vida permitió establecer el patrón usual de movilidad de una persona durante el mes anterior a la evaluación (Baker, et al., 2003). Según Peel, et al. (2005) a través de la evaluación del espacio de vida se determina el espacio donde la persona habitualmente se mueve. Curcio et al. (2013f) establecieron la validez y confiabilidad del cuestionario en adultos mayores de Colombia y Brasil. En el presente estudio se utilizó el cuestionario de la evaluación del espacio de vida (LSA) (Curcio et al., 2013) a cada adulto mayor se le preguntó:

- A. Nivel del espacio de vida: ¿Durante las últimas 4 semanas, usted ha estado en...?
1. Otras habitaciones de su casa, además de la habitación donde duerme.

2. En un área fuera de su casa como su terraza o patio, el pasillo de su edificio, garaje, en su propio jardín o en la entrada de su casa.

3. En sitios de su vecindario (barrio) que no sean su propio jardín o edificio de apartamentos.

4. Sitios fuera de su vecindario (otros barrios), pero dentro de la ciudad.

5. Sitios fuera de su ciudad.

- B. Frecuencia en términos de: ¿Cuántas veces se mueve en ese espacio?

1. Menos de 1 vez a la semana.

2. 1 a 3 veces semana.

3. 4 a 6 veces semana.

4. Diario.

- C. Independencia cuanto a: ¿ha usado ayudas o equipo? ¿Ha necesitado la ayuda de otra persona?

1. Ayuda de otro.

2. Equipo solamente.

3. Ninguno (ni ayuda personal, ni equipo).

Para la calificación del espacio de vida se utilizó el sistema original propuesto Baker et al. (2003). Se calificaron las puntuaciones para cada nivel al multiplicar el número de cada nivel (1 a 5) por el valor que se obtuvo en la independencia ( $2=$ Ninguno (ni ayuda personal, ni equipo), 1,5 = equipo solamente, $1=$ ayuda de otro) por el valor de la frecuencia de las veces que lo hace $(1=$ menos de una vez por semana, 2=1-3 veces por semana, 3= 4-6 veces por semana, 4= Diario). El espacio de vida total (LS-C) se obtiene de la multiplicación en cada uno de los 5 niveles y luego el total se da por la suma de los 5 niveles, las puntuaciones van de 0 (totalmente confinado a la cama) a 120 (viaja con frecuencia fuera de la ciudad completamente independiente).

El espacio de vida estableció tres sub escalas de acuerdo con los resultados: espacio de vida máximo (LS-M) es el nivel más alto que alcanzó, incluso cuando fue usado un equipo o la ayuda de una persona, el espacio de vida independiente (LS-I) es el nivel más alto que alcanzó sin la ayuda de una persona y sin utilizar ningún equipo y por último, el espacio de vida asistido (LS-E) es el nivel más alto que alcanzó sin la ayuda de una persona, incluso si usa el equipo. La calificación para cada nivel LS-M, LS-I y LS-E va de 0 5 , con puntuaciones más altas, mayores son los niveles de espacio de vida. Los resultados se obtuvieron de acuerdo con el software original (proporcionado por los autores de LSA).

Las características del entorno del barrio se evaluaron con una versión modificada de "The Home And Community Environment (HACE) instrument” (Keysor, Jette, Haley, 2006) que incluye los elementos construidos para la movilidad y el transporte. El instrumento tiene validez de constructo y confiabilidad comprobadas (IMIAS). Para establecer los elementos construidos para la movilidad y el transporte, se preguntó ¿En qué medida su barrio tiene...? A. Elementos construidos para la movilidad en el barrio: aceras o andenes irregulares, parques y áreas para caminar de fácil acceso, parques y áreas para caminar seguros, sitios para sentarse y descansar en las paradas del bus o en parques. Las opciones de respuesta son muchos $=1$, algunos $=2$, ninguno $=3$ y no sabe $=4$. La calificación es de $0=$ no hay obstáculos en el barrio y $1=$ sí, existen obstáculos en el barrio, puntuaciones más altas indican más obstáculos. B. 
Los elementos en el transporte: transporte público cerca a su casa, transporte público para personas con discapacidad y parqueaderos adecuados para personas con discapacidad. Las opciones de respuesta son muchos $=1$, algunos $=2$, ninguno $=3$ y no sabe $=4$. La calificación va de 1= si hay más oportunidades de transporte y $0=$ no hay oportunidades de transporte.

En un primer momento se realizó un análisis descriptivo de cada una de las variables, a continuación un análisis de la relación del espacio de vida, cruzándola con las variables socio-demográficas y características del entorno del barrio (elementos construidos, transporte y situaciones en el barrio), se aplicó la prueba Chi-cuadrado con una significancia de $(p<0,05)$. El análisis de los datos fue realizado en el programa informático Statistical Package for the Social Sciences SPSS 20.0 para Windows.

\section{RESULTADOS}

\section{Aspectos demográficos}

En la Tabla 1, se muestran los aspectos demográficos de la población estudiada, la edad se dividió en dos rangos, de 65 a 69 y de 70 a 74 , hay mayor porcentaje de ancianos en el grupo de menor edad. Respecto al estado civil, un poco más de la mitad son casados. En cuanto al nivel educativo, la mayoría de la población estudió solamente hasta primaria o menos. Un poco más de la mitad de las personas recibieron un ingreso económico mensual menor o igual a un salario mínimo legal vigente. El estrato socioeconómico de residencia predominante fue el 3.

\begin{tabular}{|c|c|c|}
\hline VARIABLE & FRECUENCIA & PORCENTAJE \\
\hline \multicolumn{3}{|l|}{ EDAD } \\
\hline 65 a 69 & 217 & 54,5 \\
\hline 70 a 74 & 181 & 45,5 \\
\hline \multicolumn{3}{|l|}{ SEXO } \\
\hline Mujer & 200 & 50,3 \\
\hline Hombre & 198 & 49,7 \\
\hline \multicolumn{3}{|l|}{ ESTADO CIVIL } \\
\hline Casados & 204 & 51,3 \\
\hline Viudos & 94 & 23,6 \\
\hline Solteros & 55 & 13,8 \\
\hline Separados / divorciados & 45 & 11,3 \\
\hline \multicolumn{3}{|l|}{ NIVEL EDUCATIVO } \\
\hline Primaria & 287 & 72,1 \\
\hline Secundaria o superior & 111 & 27,9 \\
\hline \multicolumn{3}{|c|}{ INGRESO ECONÓMICO MENSUAL } \\
\hline$\leq 1 \mathrm{SMLV}$ & 215 & 54,0 \\
\hline$\geq 2$ SMLV & 183 & 46,0 \\
\hline \multicolumn{3}{|c|}{ ESTRATO SOCIOECONÓMICO DE RESIDENCIA } \\
\hline 1 & 15 & 3,8 \\
\hline 2 & 48 & 12,1 \\
\hline 3 & 219 & 55,0 \\
\hline 4 & 62 & 15,6 \\
\hline 5 & 39 & 9,8 \\
\hline 6 & 15 & 3,8 \\
\hline
\end{tabular}

24 > Revista Márgenes No 19 Vol 13 > Diciembre 2016: 21 a 31 Facultad de Arquitectura > Universidad de Valparaíso 


\section{ESPACIO DE VIDA}

Nivel del espacio de vida, como se muestra en el Gráfico 1, más de la cuarta parte de adultos mayores se mueve fuera del barrio y en menor cantidad fuera de la ciudad.

\section{Frecuencia en el nivel espacio de vida}

\begin{tabular}{|c|c|c|c|c|c|c|c|}
\hline \multirow{2}{*}{\multicolumn{2}{|c|}{$\begin{array}{l}\text { FRECUENCIA } \\
\text { CASA }\end{array}$}} & \multicolumn{5}{|l|}{ NIVEL } & \multirow{3}{*}{$\begin{array}{l}\text { TOTAL } \\
161\end{array}$} \\
\hline & & \multirow{2}{*}{$\begin{array}{l}\text { FUERA } \\
\text { CASA } \\
2\end{array}$} & \multirow{2}{*}{$\begin{array}{l}\text { BARRIO } \\
4\end{array}$} & \multirow{2}{*}{$\begin{array}{l}\text { FUERA } \\
\text { BARRIO }\end{array}$} & \multirow{2}{*}{$\begin{array}{l}\text { FUERA } \\
\text { CIUDAD }\end{array}$} & \multirow[b]{2}{*}{76} & \\
\hline Menos de & $\mathrm{F}$ & & & & & & \\
\hline $\begin{array}{l}1 \text { vez a la } \\
\text { semana }\end{array}$ & $\%$ & $0,5 \%$ & $1,0 \%$ & $4,7 \%$ & $17,3 \%$ & $93,8 \%$ & $10,0 \%$ \\
\hline \multirow{2}{*}{$\begin{array}{l}1 \text { a } 3 \\
\text { veces } \\
\text { semana }\end{array}$} & $\mathrm{F}$ & 2 & 16 & 47 & 141 & 5 & 211 \\
\hline & $\%$ & $0,5 \%$ & $4,0 \%$ & $12,3 \%$ & $40,1 \%$ & $6,2 \%$ & $13,1 \%$ \\
\hline \multirow{2}{*}{$\begin{array}{l}4 \text { a } 6 \\
\text { veces } \\
\text { semana }\end{array}$} & $\mathrm{F}$ & 3 & 13 & 27 & 46 & 0 & 89 \\
\hline & $\%$ & $0,8 \%$ & $3,3 \%$ & $7,1 \%$ & $13,1 \%$ & $0,0 \%$ & $5,5 \%$ \\
\hline \multirow{2}{*}{ Diario } & $\mathrm{F}$ & 391 & 363 & 290 & 104 & 0 & 1148 \\
\hline & $\%$ & $98,2 \%$ & $91,7 \%$ & $75,9 \%$ & $29,5 \%$ & $0,0 \%$ & $71,3 \%$ \\
\hline \multirow{2}{*}{ TOTAL } & $\mathrm{F}$ & 398 & 396 & 382 & 352 & 81 & 1609 \\
\hline & $\%$ & $100 \%$ & $100 \%$ & $100 \%$ & $100 \%$ & $100 \%$ & $100 \%$ \\
\hline
\end{tabular}

$>$ Tabla 2. Frecuencia en el nivel espacio de vida.

En la Tabla 2 se muestra la frecuencia en días a la semana en que el adulto mayor se mueve a través de los diferentes espacios, la gran mayoría se mueve a diario en la casa y fuera de la casa, seguido de barrio y en menor proporción fuera del barrio. Se evidenció una asociación altamente significativa entre las dos variables relacionadas (Chi-cuadrado $=1.2008 .100$ y Significancia $=0,000$ ).

\section{Independencia en el nivel espacio de vida}

\begin{tabular}{|c|c|c|c|c|c|c|}
\hline \multirow{2}{*}{\multicolumn{2}{|c|}{$\begin{array}{l}\text { INDEPENDENCIA } \\
\text { CASA }\end{array}$}} & \multicolumn{5}{|l|}{ NIVEL } \\
\hline & & \multirow{2}{*}{$\begin{array}{l}\text { FUERA } \\
\text { CASA } \\
0\end{array}$} & \multirow{2}{*}{$\begin{array}{l}\text { BARRIO } \\
2\end{array}$} & \multirow{2}{*}{$\begin{array}{l}\text { FUERA } \\
\text { BARRIO } \\
17\end{array}$} & \multirow{2}{*}{$\begin{array}{l}\text { FUERA } \\
\text { CIUDAD }\end{array}$} & \multirow[b]{2}{*}{317} \\
\hline No se mueve & $f$ & & & & & \\
\hline en este nivel & $\%$ & $0,0 \%$ & $0,5 \%$ & $4,2 \%$ & $11,6 \%$ & $79,6 \%$ \\
\hline \multirow{2}{*}{$\begin{array}{l}\text { Con ayuda de } \\
\text { otro }\end{array}$} & $f$ & 21 & 22 & 19 & 19 & 2 \\
\hline & $\%$ & $5,3 \%$ & $5,5 \%$ & $4,8 \%$ & $4,8 \%$ & $0,5 \%$ \\
\hline \multirow{2}{*}{ Sin ayuda } & $f$ & 377 & 374 & 362 & 333 & 79 \\
\hline & $\%$ & $94,7 \%$ & $94,0 \%$ & $91,0 \%$ & $83,7 \%$ & $19,8 \%$ \\
\hline \multirow{2}{*}{ TOTAL } & $f$ & 398 & 398 & 398 & 398 & 398 \\
\hline & $\%$ & $100 \%$ & $100 \%$ & $100 \%$ & $100 \%$ & $100 \%$ \\
\hline
\end{tabular}

$>$ Tabla 3. Independencia en el nivel espacio de vida

Como se muestra en la Tabla 3, la mayoría de los adultos mayores del estudio se mueve sin la ayuda de una persona fuera del barrio y en menor proporción fuera de la ciudad. El estadístico Chi-cuadrado de la tabla de contingencia anterior, se muestra que sí hay diferencias significativas entre los valores de las categorías (Chicuadrado $=1,195$ y Significancia $=0,000$ ).

Espacio de vida total, es la medida compuesta por nivel alcanzado, independencia y frecuencia, la media de las puntuaciones fue 62,51 . La población de estudio que se encuentra por debajo del punto de corte (56 puntos) presentó el $26,9 \%$ de riesgo de limitación de las actividades instrumentales de la vida diaria.
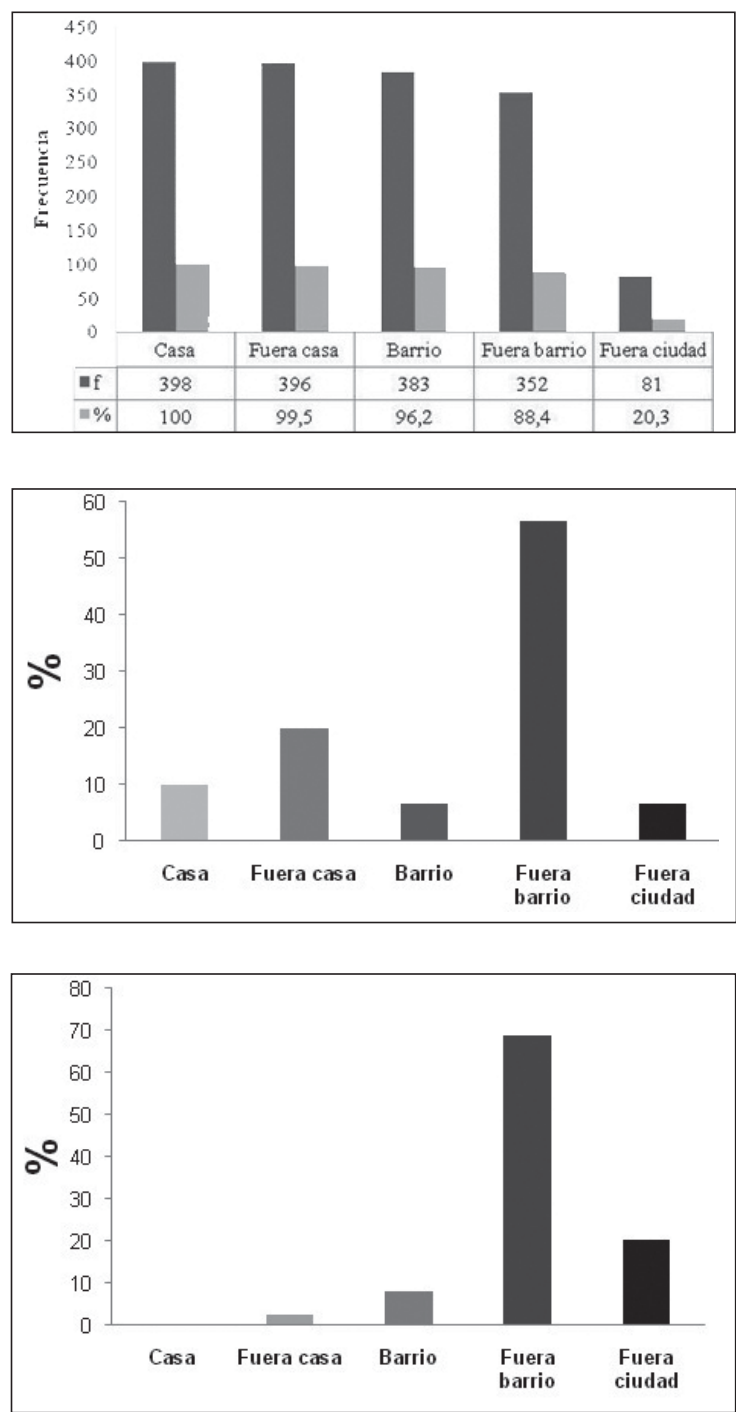

$>$ Gráfico 1. Nivel espacio de vida.

$>$ Gráfico 2. Espacio de vida máximo.

$>$ Gráfico 3. Espacio de vida independiente. 
En el espacio de vida total se establecieron tres sub escalas de acuerdo con los resultados: Espacio de vida máximo, en el gráfico 2, el espacio de vida máximo, el nivel más alto que alcanzaron los adultos mayores cuando requieren la ayuda de una persona, en mayor proporción fue fuera del barrio y en menor proporción fuera de la ciudad.

En el Gráfico 3, Espacio de vida independiente, el nivel más alto que alcanzaron los adultos mayores sin la ayuda de una persona, en mayor proporción fue fuera del barrio y en menor proporción fuera de la ciudad.

Espacio de vida asistido, el 92,5\% de los adultos mayores del estudio alcanzaron el nivel más alto sin la ayuda de una persona y el $7,5 \%$ de los adultos mayores requirieron la ayuda de una persona para moverse en un nivel del espacio de vida.

Espacio de vida total y aspectos demográficos, El espacio de vida total se asocia significativamente $(p=0,000)$ con el sexo, nivel educativo e ingreso económico mensual. El espacio de vida independiente se asocia significativamente $(p=0,000)$ con el ingreso económico mensual. A un mayor espacio de vida mayor ingreso económico.

\section{ENTORNO DEL BARRIO}

En la Tabla 4 se presentan las características en el entorno del barrio en cuanto a los elementos construidos. Más de la mitad de los ancianos consideraron que son un obstáculo para el desplazamiento las aceras o andenes irregulares, los parques o áreas para caminar de fácil acceso y los parques o áreas para caminar seguros; en cuanto a los sitios para sentarse o descansar en la parada del bus o en parques, menos de la mitad no lo consideraron como un obstáculo, con diferencias significativas ( $p=0,000)$, por lo que se puede decir que la percepción de los ancianos frente a los obstáculos propuestos en el presente estudio no es homogénea.

\begin{tabular}{|c|c|c|c|c|}
\hline \multicolumn{5}{|l|}{ VARIABLES } \\
\hline ELEMENTOS CONSTRUIDOS & \multicolumn{2}{|c|}{ Sí ES UN OBSTÁCULO } & \multicolumn{2}{|c|}{ NO ES UN OBSTÁCULO } \\
\hline Aceras o andenes irregulares & 291 & $73 \%$ & 107 & $27 \%$ \\
\hline Parques y áreas para caminar fácil acceso & 237 & $60 \%$ & 161 & $40 \%$ \\
\hline Parques y áreas para caminar seguro & 218 & $55 \%$ & 180 & $45 \%$ \\
\hline Sitios para sentarse y descansar & 184 & $46 \%$ & 214 & $54 \%$ \\
\hline TRANSPORTE PÚBLICO & \multicolumn{2}{|c|}{ SÍ HAY OPORTUNIDADES } & \multicolumn{2}{|c|}{ NO HAY OPORTUNIDADES } \\
\hline Transporte público cerca casa & 370 & $93 \%$ & 28 & $7 \%$ \\
\hline Transporte público para personas con discapacidad & 28 & $7 \%$ & 370 & $93 \%$ \\
\hline Parqueaderos adecuados para personas con discapacidad & 8 & $2 \%$ & 390 & $98 \%$ \\
\hline SITUACIÓN EN EL BARRIO & \multicolumn{2}{|c|}{ SÍ ES UN PROBLEMA } & \multicolumn{2}{|c|}{ NO ES UN PROBLEMA } \\
\hline Tensiones grupos raciales étnicos o religiosos & 56 & $14 \%$ & 342 & $86 \%$ \\
\hline Basuras desperdicios o botellas en calle & 190 & $48 \%$ & 208 & $52 \%$ \\
\hline Venta o uso drogas & 158 & $40 \%$ & 240 & $60 \%$ \\
\hline Abuso alcohol calles y sitios públicos & 151 & $38 \%$ & 247 & $62 \%$ \\
\hline Pandillas & 142 & $36 \%$ & 256 & $64 \%$ \\
\hline Iluminación inadecuada o insuficiente & 93 & $23 \%$ & 305 & $77 \%$ \\
\hline Delincuencia - delitos & 215 & $54 \%$ & 183 & $46 \%$ \\
\hline Ruido excesivo & 160 & $40 \%$ & 238 & $60 \%$ \\
\hline Tráfico pesado & 163 & $41 \%$ & 235 & $59 \%$ \\
\hline Casas o edificios abandonados & 38 & $10 \%$ & 360 & $90 \%$ \\
\hline
\end{tabular}

26 > Revista Márgenes № 19 Vol 13 > Diciembre 2016: 21 a 31 Facultad de Arquitectura > Universidad de Valparaíso 
En relación al transporte público, en la Tabla 4 se muestra que casi la totalidad de los ancianos percibieron que no existen parqueaderos adecuados y transporte público para personas con discapacidad, por otra parte, casi la misma proporción manifestaron que si hay transporte público cerca a su casa. En resumen, sí hay transporte disponible, pero no está adecuado a las necesidades de los ancianos con discapacidad.

Respecto a la situación en el barrio, en la Tabla 4 se muestra que más de la mitad de los ancianos consideraron que sí es un problema serio la delincuencia y los delitos, seguido de basuras, desperdicios o botellas rotas en la calle, en los andenes o en los jardines. En síntesis, las diferentes situaciones en el barrio expuestas en la entrevista no se perciben de igual forma por los ancianos, existieron algunos problemas más serios que otros $(p=0,000)$.

\section{ENTORNO DEL BARRIO Y NIVELES DE ESPACIO DE VIDA}

Los elementos construidos del barrio (parques y áreas para caminar seguro - parques y áreas para caminar de fácil acceso) se asocian significativamente $(p<0,05)$ con los niveles de espacio de vida en los adultos mayores del estudio (Tabla 5 ).

\begin{tabular}{|c|c|c|}
\hline VARIABLES & CHI-CUADRADO & SIGNIFICANCIA \\
\hline \multicolumn{3}{|l|}{ ELEMENTOS CONSTRUIDOS } \\
\hline Parques y áreas para caminar seguro & 9,310 & 0,010 \\
\hline Parques y áreas para caminar fácil acceso & 6,015 & 0,049 \\
\hline Aceras o andenes irregulares & 5,083 & 0,079 \\
\hline Sitios para sentarse y descansar & 1,320 & 0,517 \\
\hline \multicolumn{3}{|l|}{ TRANSPORTE PÚBLICO } \\
\hline Transporte público para personas con discapacidad & 2,432 & 0,296 \\
\hline Parqueaderos adecuados para personas con discapacidad & 1,773 & 0,412 \\
\hline Transporte público cerca casa & 1,302 & 0,522 \\
\hline \multicolumn{3}{|l|}{ SITUACIÓN EN EL BARRIO } \\
\hline Delincuencia - delitos & 4,372 & 0,112 \\
\hline Tensiones grupos raciales étnicos o religiosos & 4,174 & 0,124 \\
\hline Ruido excesivo & 3,599 & 0,165 \\
\hline Iluminación inadecuada o insuficiente & 3,495 & 0,174 \\
\hline Basuras desperdicios o botellas en calle & 3,032 & 0,220 \\
\hline Venta o uso drogas & 1,499 & 0,472 \\
\hline Casas o edificios abandonados & 1,468 & 0,480 \\
\hline Tráfico pesado & 0,669 & 0,716 \\
\hline Abuso alcohol calles y sitios públicos & 0,518 & 0,772 \\
\hline Pandillas & 0,377 & 0,828 \\
\hline
\end{tabular}

> Tabla 5. Características entorno del barrio y niveles de espacio de vida.

\section{DISCUSIÓN}

Los hallazgos de este estudio reflejan que la mayoría de la población sale fuera de la casa, sin la ayuda de una persona y a diario, esto evita la restricción en el espacio de vida, por ende trae beneficios para la movilidad, resultados similares se encuentran en varios estudios (Shimida, et al., 2013; Peel, et al., 2005). Cuando las personas obtienen una mayor puntuación se mueven en lugares que van desde la casa hasta fuera del barrio (71,94 puntos). 
Respecto al nivel educativo, se considera que desempeña un papel muy importante en relación a cómo se vivirá al ser adulto mayor (Gómez Montes \& Curcio Borrero, 2002), la experiencia educativa contribuye a la alfabetización (Manly, Touradji, Tang, \& Stern, 2003), en si aprender a escribir y leer, permite tener más oportunidades en la vida. En ese sentido el nivel educativo es un proceso continuo de organización y construcción de la experiencia en relación a lo que se aprende y aquello que coloca en práctica en la vida diaria, en la medida que lo prepara para anticipar lo que puede ocurrir y, en resultado, dirigir sus acciones a solucionar un problema (De la Barrera, Donolo, \& Rinaudo, 2010).

Los hallazgos de este estudio en lo que respecta al nivel de educación y un entorno con obstáculos en los elementos construidos del barrio, se corrobora con varios estudios en donde proponen que una relación entre nivel de educación (Elizondo-Armendáriz, Guillén Grima \& Aguinaga Ontoso, 2005) y un entorno con obstáculos (Rantanen, et al., 2013) requiere de las personas resolver problemas, ayuda del otro, toma de decisiones y desafíos, el cual puede ser un contexto para un mayor nivel de actividad física, lo cual está comprobado beneficia la salud del adulto mayor (Ludlow \& Roth, 2011).

El espacio de vida (Baker et al., 2003:1614) permite conocer el lugar en que se mueve una persona, a la vez moverse por sí mismo dentro de los entornos de la comunidad (Webber et al., 2010). Los adultos mayores que se mueven de forma independiente en el área urbana de Manizales, requieren moverse a través de los diferentes espacios, debido a los desafíos que ocasiona la topografía de la ciudad. También se encuentran resultados similares en varios estudios (Rantannen, et al., 2013; Allman, et al., 2004). Es importante precisar que utilizar el transporte público para moverse independientemente en los espacios de la ciudad, requiere un mayor movimiento (Davis, et al., 2011), esto a la vez proporciona oportunidades para la participación de los adultos mayores en la comunidad. También el espacio de vida independiente favorece el envejecimiento activo (OMS, 2002b) de las personas durante más tiempo.

En cuanto al espacio de vida y los aspectos demográficos, los resultados coinciden con los de otros estudios a nivel local (Curcio, et al., 2013) e internacional (Snih, et al., 2012; Baker, et al., 2003) que muestran una asociación significativa entre el espacio de vida total y sexo, nivel educativo e ingreso económico. Las puntuaciones bajas en el espacio de vida se presentan en mujeres, con un nivel de primaria o menos y menores ingresos económicos para cubrir las necesidades básicas.

Para moverse en los niveles del espacio de vida, un adulto mayor requiere de la capacidad funcional y también sortear los desafíos que demanda la vida diaria al estar en un entorno, de allí, que el nivel en que más se mueve la población del estudio es fuera del barrio, salir de este nivel requiere que la persona utilice el transporte público o privado (Gómez Montes \& Curcio Borrero, 2014), por ende moverse fuera de la ciudad implica una mayor funcionalidad y recurso económico.

Respecto a la frecuencia en el espacio de vida, estudios establecen que los adultos mayores que con más frecuencia se mueven en un lugar del espacio de vida se protegen contra la fragilidad y discapacidad (Kono, et al., 2007; Fujita, et al., 2006). Esto hace pensar que una gran proporción de la población del presente estudio, de acuerdo a la frecuencia del espacio de vida, puede estar protegida contra la fragilidad y discapacidad, es decir, el hecho que la población del estudio se mueve con más frecuencia fuera de la casa afronta los retos que implica salir de ella, salir más y con menos ayuda a otros lugares del área urbana de Manizales. A la vez, salir fuera de la ciudad está determinado por el ingreso económico, por ello si el ingreso es bajo restringe la frecuencia (Rosso, et al., 2013).

La independencia se puede entender como la capacidad de realizar las tareas y asumir los roles sociales que demanda la vida diaria (Gómez Montes \& Curcio Borrero, 2002), la limitación funcional, se refiere a la necesidad de ayuda de una persona para moverse en diferentes lugares. En la población del estudio la movilidad real sin la ayuda de una persona se presenta en la gran mayoría hasta el nivel fuera del barrio. Se comprende entonces que los adultos mayores tienen un buen funcionamiento, lo cual permite alcanzar el nivel más alto en el espacio de vida. Por otro lado, una minoría de los adultos mayores que presentan limitaciones funcionales necesitan de la ayuda de una persona para alcanzar el nivel más alto en el espacio de vida.

Tal como se mencionó, en el espacio de vida al combinar nivel alcanzado, independencia y frecuencia se obtiene la puntuación total, por lo cual una mayoría de la población del estudio se encuentra por encima del punto de corte de 56 (Shimada, et al., 2010). Las personas que se encuentran por encima del punto de corte son independientes en la casa, se mueven en el barrio y fuera de él, por lo general van fuera de la ciudad. La población que se encuentra por debajo del punto de corte (Shimada, et al., 2010) deben ser considerados para integrar un programa de intervención, el cual puede beneficiar su movilidad en los lugares de espacios de vida. En el estudio de Curcio, et al. (2013), realizado en Manizales, el punto de corte se establece en 51,8. Hallazgo diferente se encuentra en la población del estudio, lo cual indica que las personas tienen puntuaciones más altas y por ende una mayor movilidad en los espacios de vida.

La población del estudio que se encuentra en el espacio de vida máximo, necesita de la ayuda de una persona para alcanzar el nivel fuera del barrio. Contrario a los hallazgos en el estudio de Curcio, et al. (2013) en donde se alcanza el nivel del barrio, pues presentan una mayor limitación en movilidad y discapacidad, a la vez estos resultados están de acuerdo con varios estudios (Rosso, et al., 2013; Shimada, et al., 2010). Se hace prioritario que los adultos mayores del estudio que necesitan de la ayuda de una persona para alcanzar el nivel más alto en el espacio de vida máximo, sean beneficiados con actividades que mantengan su independencia fuera de la casa.

En el espacio de vida independiente, la mayoría de población alcanza el nivel fuera del barrio. Contrasta con los hallazgos en el estudio de Curcio, et al. (2013) donde se alcanza el nivel del barrio, pues presenta una limitación en movilidad y discapacidad. El alcanzar un nivel más alto en el espacio de vida independiente permite a la población de estudio realizar tareas y asumir roles sociales sin la asistencia de una persona, tal como se ha demostrado en varios estudios (Webber, et al., 2010; OMS, 2007).

En relación al espacio de vida asistido la mayoría de la población del estudio alcanza sin la ayuda de una persona el nivel más alto. Estos hallazgos están de acuerdo con varios estudios (Rosso, et al., 2013; Webber, et al., 2010) en la cual establecen que los adultos mayores independientes desempeñan las funciones de la vida diaria y viven en la comunidad con poca ayuda o ninguna. 
El entorno del barrio juega un papel fundamental en la configuración del área urbana de Manizales, en estrecha relación entre el paisaje de montaña y las construcciones. La ciudad está ubicada en la cordillera central de los Andes Colombianos; tiene una alta influencia tectónica y volcánica, y esto ocasiona que la topografía sea diversa desde zonas abruptas y escarpadas hasta valles amplios (Plan de Ordenamiento Territorial, 2008). Es por esto que en los barrios la construcción de los elementos como las aceras o andenes se condiciona por las pendientes $\geq 15^{\circ}$ que se forman en el área urbana (Plan de Ordenamiento Territorial, 2008; Fique, et al., 2000). Este estudio corrobora los hallazgos de un estudio local (Curcio, et al., 2013) en cuanto que las aceras o andenes son obstáculos para la movilidad de los adultos mayores, debido a la presencia de las irregularidades en los materiales, específicamente en la textura del piso (cemento, baldosa), acabado del piso (grietas o baches) y cambios de nivel (pendiente) (Anexo 6 Características aceras y materiales). Estas irregularidades en las aceras o andenes pueden afectar más a los adultos mayores que requieren la ayuda de una persona para moverse en un lugar en el barrio (Clarke et al., 2011) y puede exponer al adulto mayor a un riesgo alto de caídas y a una restricción a la casa (Gómez Montes, 2011; Paterson \& Warburton, 2010).

La mayoría de la población del estudio considera que en Manizales, los parques o áreas para caminar en el barrio, presentan un obstáculo para el fácil acceso y no son seguros respecto a los elementos que componen el mobiliario, resultados similares se encontraron en el estudio de Rantanen (2013). El desplazamiento de los adultos mayores del estudio a estos lugares presentan dificultad de acceso, principalmente por el número limitado de parques (199), lo cual arroja un resultado de un parque por 2.000 habitantes, también los metros ${ }^{2}(1,1)$ por habitante están por debajo de ciudades en Colombia (Bogotá, Bucaramanga, Medellín y Pereira) y a nivel internacional (Buenos Aires, Santiago de Chile) (Plan de Ordenamiento Territorial POT 2008 - 2011).

La topografía del área urbana condiciona la accesibilidad (OMS, 2007) a los parques y áreas para caminar debido a la ubicación en vías de tráfico rápido, amoblado no adaptado al usuario y tipo de construcción: diseño, tamaño, franja de circulación y pocos espacios para adultos mayores. Según Rantanen (2013) una disminución de los obstáculos en los elementos construidos puede mejorar la accesibilidad de los adultos mayores a estos lugares.

En suma, la adaptación de la persona al ambiente, según Lawton (1975), es dada por la exigencia desde el exterior (características de cada elemento construido en el barrio) al interactuar con el adulto mayor. Sin embargo, hay que precisar que los obstáculos o dificultades para la movilidad, según Rantanen, et al. (2013) afectan más a los adultos mayores que tienen mayor dependencia, pues los independientes deben interactuar en la vida diaria con los obstáculos, movilizarse de un lugar a otro en el barrio y realizar actividades físicas, entre ellas caminar, subir - bajar escaleras. Esto se corrobora en el estudio, pues la mayoría de los adultos mayores son independientes.

Respecto al transporte público cerca a la casa, éste desempeña un papel importante para la realización de actividades de la vida diaria (Zeitler, et al., 2012; Talbot, et al., 2004), la población del estudio considera que sí hay oportunidades de transporte público, esto corrobora lo expuesto en el informe del POT (2008 - 2011), en el cual, el número de vehículos de transporte (buses, busetas, colectivos y taxis) por millón de habitantes es de 2.203. Esta cifra supera ampliamente los estándares latinoamericanos de transporte público. Otra característica importante del transporte público es el uso (Chaudhury, et al., 2012), y está determinado por la existencia de densidad habitacional alta en el área urbana de Manizales.

El hallazgo de la falta de oportunidades en parqueaderos y transporte público para discapacitados ha sido reportado previamente en la literatura (White et al., 2010). Dicho hallazgo también se reportó en este estudio. En Manizales, la ausencia de parqueaderos se fundamenta en las condiciones naturales del suelo y la topografía, la cual condiciona la franja de terreno para la construcción de parqueaderos en las vías de la ciudad, y queda relegado a la construcción privada (Propiedad horizontal habitacional y comercial) y en el transporte público para personas discapacitadas se establece en el incumplimiento en los estándares de calidad y seguridad (no adecuación en el equipamiento de transporte colectivo: estaciones, terminales de transporte urbano, depósitos de buses, busetas, colectivos y parqueaderos públicos), los vehículos no cuentan con pisos que desciendan, escalones bajos, asientos amplios y altos. White, et al. (2010) plantean que la falta de parqueaderos y transporte público para discapacitados puede interferir en el desarrollo de los encuentros sociales (familiares, amigos y organización formal) y en la actividad física.

Al igual que en otros estudios a nivel local (Curcio et al., 2013) e internacional (Sánchez - González, 2009) en este estudio, se corrobora que la existencia de delincuencia - delitos son un problema serio en el barrio que afectan al adulto mayor (Yen et al., 2009).

La seguridad en el barrio se percibe de acuerdo a como se vive la vida diaria (Farrall, Jackson, \& Gray, 2008;) y afecta la predisposición de los adultos mayores a moverse en el barrio, la pérdida de la seguridad puede ser producto de un proceso silencioso o abrupto contra alteraciones súbitas que afectan las actividades y encuentros sociales dados en el barrio. La seguridad personal (Ballesteros Arjona, V., 2011; Segovia \& Jordán, 2005) es tan fundamental respecto de la violencia física (amenaza de individuos). En este sentido los adultos mayores del estudio se sienten amenazados en mayor proporción en el espacio físico del barrio (calles, parques y áreas para caminar).

\section{AGRADECIMIENTOS}

IMIAS tiene apoyo financiero de The Canadian Institutes of Health Research (CIHR), y colaboración de las universidades Université de Montreal, Quebec, Canadá; Universidade Federal do Rio Grande do Norte, Brasil; Queens University, Kingston, Canadá; Universidad de CaldasManizales, Colombia e Instituti i Shendetit Publik, Tirana, Albania.

\section{BIBLIOGRAFÍA}

ALCALDÍA DE MANIZALES (2007) "Plan de Ordenamiento Territorial de Manizales", Manizales: Secretaria de Planeación Municipal.

ALLMAN, R. M. et al. (2004) "Racial similarities and differences in predictors of mobility change over eighteen months", Journal of General Internal Medicine 19, pp. 1118-1126.

BAKER, P. S., BODNER, E. V. \& ALLMAN, R. M. (2003) “Measuring life space mobility in community-dwelling older adults", Journal of the American Geriatrics Society 51(11), pp.1610-1614. 
BALFOUR, J. J. \& KAPLAN, G. A. (2002) "Neighborhood environment and loss of physical function in older adults: evidence from the Alameda County Study", American Journal of Epidemiology 155(6), pp. 507-515.

BALLESTEROS ARJONA, V. (2011) Urbanismo, medio ambiente y salud, Andalucía: Escuela Andaluza de Salud Pública.

BRONFENBRENNER, U. (1987) La ecología del desarrollo humano, Barcelona: Paidós Ibérica.

DAVIS, M. G., FOX, K. R., HILLSDON, M., COULSON, J. C., SHARP, D. J., STATHI, A. y otros (2011) "Getting out and about in older adults: the nature of daily trips and their association with objectively assessed physical activity", International Journal Behavior Nutrition Physical Activity 8(1), p. 116.

CLARKE, P. J. et al. (2011) "Participation among Adults with Disability: The Role of the Urban Environment”, Social Science Medicine 72(10), pp. 1674-1684.

CLARKE, P. \& NIEUWENHUIJSEN, E. R. (2009) "Environments for healthy ageing: A critical review”, Maturitas 64, pp. 14-19.

CROWE, M., et al. (2008) "Life-Space and cognitive decline in a community-based sample of African American and Caucasian older adults", Journals Gerontology American Biology Science Medical Sciences 63(11), pp. 1241-1250.

CURCIO, C. L., ALVARADO, B. E., GÓMEZ, J. F., GUERRA, R., GURALNIK, J., \& ZUNZUNEGUI, M. V. (2013) “Life Space assessment scale to assess mobility: validation in Latin American elderly women and men", Aging Clinical Experimental Research 25(5), pp. 553-560.

DANE (2005) Censo General 2005, Bogotá: Departamento Administrativo Nacional de Estadística.

DE LA BARRERA, M. L., DONOLO, D. \& RINAUDO, M. C. (2010) "Riesgo de demencia y niveles de educación: Cuando aprender es más saludable de lo que pensamos", Anales de psicología 26(1), pp. 34-40.

FARRALL, S., JACKSON, J. \& GRAY, E. (2008) “La transcendencia cultural y social de la inseguridad ante la delincuencia", en A. Serrano Maillo \& J. L. Guzmán Dalbora, Procesos de infracción de normas y de reacción a la infracción de normas, pp. 233-276, Madrid: Dykinson.

FIQUE, L., CERÓN, D. \& ROJAS, A. M. (2000) Accesibilidad al medio físico y al transporte. Manual de referencia, Bogotá: Universidad Nacional de Colombia.

FUJITA, K., FUJIWARA, Y., CHAVES, P. H., YUTAKA, M. \& SHOJI, S. (2006) "Frequency of Going Outdoors as a Good Predictors for Incident Disability of Physical Function as well as Disability Recovery in Community-Dwelling Older Adults in Rural Japan", Journal of Epidemiology 16(6), pp. 261-270.

FRANK, L., KERR, J., ROSENBERG, D. \& KING, A. (2010) “Healthy Aging and Where You Live: Community Design Relationships With Physical Activity and Body Weight in Older Americans", Journal of Physical Activity and Health 7(1), pp. 82-90.
GARCÍA DE YÉBENES, M. J., RODRÍGUEZ LASO, A., OTERO PUIME, A., ZUNZUNEGUI, M. V. \& DEL SER, T. (2003) "Validation of a short cognitive tool for the screening of dementia in low educated elderly people", International Journal Geriatric Psychiatry 18, pp. 926-936.

GÓMEZ MONTES, J. F. (2011c) Caídas en ancianos, Manizales: Universidad de Caldas.

GÓMEZ MONTES, J. F. \& CURCIO BORRERO, C. L. (2014) Salud del anciano: valoración, Manizales: Asociación Colombiana de Gerontología y Geriatría.

GÓMEZ MONTES, J. F. \& CURCIO BORRERO, C. L. (2011) Manual de procedimientos para el International Mobility in Aging Study, Manizales: Grupo investigaciones en gerontología y geriatría Universidad de Caldas.

GÓMEZ MONTES, J. F. \& CURCIO BORRERO, C. L. (2002) Valoración integral de la salud del anciano, Manizales: Tizan.

HUENCHUAN, S. (2009) “Envejecimiento, derechos humanos y políticas públicas”, pp. 1-232, Santiago de Chile: Naciones Unidas.

KONO, A., KAI, I., SAKATO, C. \& RUBENSTEIN, L. Z. (2007) “Frequency of going outdoors predicts long-range functional change among ambulatory frail elders living at home", Archives Gerontology Geriatric 45(3), pp. 233-242.

KEYSOR, J. J. \& JETTE, A. M. (2005) "Development of the home and community environment", Journal Rehabilitation Medicine 37 , pp. $37-44$.

KEYSOR, J. J., JETTE, A. M., BETTGER, J. P. \& HALEY, S. M. (2006) "Association of environmental factors with levels of home and community participation in an adult rehabilitation cohort”, Archives Physical Medicine Rehabilitation 87, pp. 1566-1574.

LAWTON, M. P. (1975) "Competence, environmental press and adaptation”, en P. G. Windley, T. O. Byerts \& G. Ernst, Theory development in environment and aging, Washigton D. C.: Gerontological Society.

LUDLOW, A. T. \& ROTH, S. M. (11.02.2011) "Physical activity and telomere biology: exploring the link with aging-related disease prevention", recuperado el 10 de Abril de 2016, de PubMed: http://www.ncbi.nlm.nih.gov/ pubmed/21403893.

OMS (2007) Global age-friendly cities: A guide, Geneva, Switzerland: World Health Organization.

OMS (12.08.2002) "Envejecimiento activo: un marco político", recuperado el 23 de Marzo de 2016, Revista española de geriatría y gerontología: http://www.doyma.es/revistas/ ctl_servlet?_f=7010\&sumarioid=13001708

ONU (2002) World Population Ageing 1950-2050 (ST/ESA/ SER.A/207), New York: Naciones Unidas, $N^{\circ}$ de venta: E.02.XIII.3.

MANLY, J., TOURADJI, P., TANG, M. X. \& STERN, Y. (2003) “Literacy and Memory Decline Among Ethnically Diverse Elders", Journal of Clinical and Experimental Neuropsychology 25, pp. $680-690$. 
PATERSON, D. H., WARBURTON, D. E. (11.05.2010) "Physical activity and functional limitations in older adults: a systematic review related to Canada's Physical Activity Guidelines", recuperado el 11 de Abril de 2016, Int. J. Behav. Nutr. Phys. Act: http://www.ncbi.nlm.nih.gov/ pubmed/20459782

PEEL, C. et al. (2005) "Assessing mobility in older adults: the UAB Study of Aging Life-Space Assessment", Journal Orthopaedic Sports Physical Therapy 85(10), pp. 1008-1119.

RANTANEN, T. (2013) "Promoting Mobility in Older People", J. Prev. Med. Public Health 46, pp. 50-54.

RENALDS, A., SMITH, T. H. \& HALE, P. J. (2010) "A systematic review of built environment and health", Family and Community Health $33(1)$, pp. 68-78.

Rosso, A. L., AUCHINCLOSS, A. H. \& MICHAEL, Y. L. (2011) “The Urban Built Environment and Mobility in Older Adults: A Comprehensive Review", Journal of Aging Research, pp. 1-10.

SÁNCHEZ-GONZÁLEZ, D. (2009) "Contexto ambiental y experiencia espacial de envejecer en el lugar: el caso de Granada", Papeles de Población 15(60), pp. 175-213.

SEGOVIA, O. \& JORDÁN, R. (2005) Espacios públicos urbanos, pobreza y construcción social, Santiago de Chile: CEPAL.

SNIH, S. A., PEEK, K. M., SAWYER, P., MARKIDES, K. S., ALLMAN, R. M. \& OTTENBACHER, K. J. (2012) "Life-space mobility in Mexican Americans aged 75 and older", Journal of the American Geriatrics Society 60(3), pp. 532-537.

SHIMADA, H., ISHIZAKI, T., KATO, M., MORIMOTO, A., TAMATE, A., UCHIYAMA, Y. y otros (2010) "How often and how far do frail elderly people need to go outdoors to maintain functional capacity?", Arch. Gerontol. Geriatr. 50(2), pp. 140-146.

SCHOOTMAN, M. et al. (2006) “Neighborhood Conditions and Risk of Incident Lower-Body Functional Limitations among Middle-aged African Americans", American Journal Epidemiology (163), pp. 450-458.

TALBOT, L. R., VISCOGLIOSI, C., DESROSIERS, J., VINCENT, C., ROUSSEAU, J. \& ROBICHAUD, L. (2004b) "Identification of rehabilitation needs after a stroke: an exploratory study”, Health Qual. Life Outcomes 2, p. 53.

WHITE, D. K. et al. (2010) "Are features of the neighborhood environment associated with disability in older adults?", Disability Rehabilitation 32(8), pp. 639-645.

WEBBER, S. C., PORTER, M. M. \& MENEC, V. H. (2010) "Mobility in older adults: A comprehensive framework", The Gerontologist 50(4), pp. 443-450.

YEN, I. H., MICHAEL, Y. L. \& PERDUE, L. (2009) “Neighborhood Environment in Studies of Health of Older Adults: A Systematic Review", American Journal Preventive Medicine 37(5), pp. 455-463.

ZEITLER, E., BUYS, L., AIRD, R. \& MILLER, E. (2012) “Mobility and Active Ageing in Suburban Environments: Findings from InDepth Interviews and Person-Based GPS Tracking" Current Gerontology and Geriatrics Research, pp. 1-20.
ZUNZUNEGUI, M. V. et al. (2000) “Development of simple cognitive function measures in a community dwelling population of elderly in Spain", International Journal Geriatric Psychiatry 15, pp. 130-140. 